Voix et Images

volxetimages

\title{
« Des lectures illicites » : une entrevue avec J.-Z.-Léon Patenaude
}

\section{Bertrand Gervais}

Volume 15, numéro 2 (44), hiver 1990

Pratiques illicites

URI : https://id.erudit.org/iderudit/200832ar

DOI : https://doi.org/10.7202/200832ar

Aller au sommaire du numéro

Éditeur(s)

Université du Québec à Montréal

ISSN

0318-9201 (imprimé)

1705-933X (numérique)

Découvrir la revue

Citer ce document

Gervais, B. (1990). « Des lectures illicites » : une entrevue avec J.-Z.-Léon

Patenaude. Voix et Images, 15(2), 167-178. https://doi.org/10.7202/200832ar d'utilisation que vous pouvez consulter en ligne.

https://apropos.erudit.org/fr/usagers/politique-dutilisation/ 


\section{«Des lectures illicites»: une entrevue avec J.-Z.-Léon Patenaude}

\section{par Bertrand Gervais, Université du Québec à Montréal}

Il n'appartient pas à des policiers ou $\grave{a}$ des douaniers de se constituer censeurs de ce que les citoyens peuvent lire ou voir. 1

Il aimait se faire appeler Monsieur Livre. Et de fait, sa vie entière a été consacrée à les publier, les présenter, les vendre et encore, à les collectionner. Il fut secrétaire général et membre honoraire de l'Association des éditeurs canadiens-français, secrétaire du Conseil supérieur du livre, responsable du Salon du livre de Montréal ainsi que du Centre de diffusion du livre canadienfrançais à Paris, fondateur de la Société du droit d'auteur au Canada, éditeur pour le gouvernement, etc. Son curriculum vitæ fait quelque cinquante pages!

Jules-Zénon-Léon Patenaude collectionnait les livres: les livres québécois, les livres sur les sociétés secrètes, la franc-maçonnerie, ainsi que sur l'érotisme et la pornographie (sa collection cochonne, comme il l'a appelée lui-même en riant). Il a fait don de ses deux collections les plus importantes, celle sur l'érotisme qui compte un peu moins de quatre cent cinquante titres et celle sur les sociétés secrètes qui en compte plus de cent cinquante, à la bibliothèque de l'Université du Québec à Montréal; ces collections constituent le fonds J.-Z.-Léon Patenaude ${ }^{2}$. Nous l'avons rencontré afin qu'il nous explique un peu comment sa collection sur l'érotisme s'est constituée. Une telle compilation présuppose des lectures quelque peu illicites!

J.-Z.-Léon Patenaude n'était pas un intellectuel, il s'est même plutôt senti rejeté par ceux-ci; il n'était pas un homme de lettres, non, simplement un homme de livres. Il est mort en juillet 1989, à 63 ans, des suites d'une longue maladie.

V. \& I. - Votre collection d'œuvres érotiques compte, selon les données de la bibliothèque de l'UQAM, quelque 430 titres, certains datant même des années 30 et 40. C'est une collection importante. À quand remonte l'idée d'une telle collection? Comment tout cela a-t-il commencé?

J.-Z.-L. Patenaude - Il faut dire que j'ai été un homme indépendant d'esprit très jeune et que très tôt, j'ai commencé à lire ce qui ne pouvait être lu. Dans les

1 Tiré d'un communiqué de presse de l'Association des éditeurs canadiens, au sujet de la saisie de deux livres des éditions Parti pris, daté du 11 novembre 1969 et signé par Jacques Hébert, président, et J.-Z.-Léon Patenaude, secrétaire administratif.

2 La plupart des livres dont il sera question ici proviennent du fonds J.-Z.-Léon Patenaude; nous ne spécifierons que la référence exacte des titres qui n'en font pas partie. 
années 45-50, deux livres faisaient autorité au Québec: c'étaient les Romans à lire et romans à proscrire de l'abbé Louis Bethléem et le Répertoire alphabétique de 10000 auteurs avec 40000 de leurs ouvrages de 1945 du père Georges Sagehomme. Ceux-là décidaient ce qui devait être lu. Moi, je me mets à lire du Zola et un certain nombre d'auteurs et d'ouvrages, dont Gamiani: ou deux nuits d'excès d'Alfred de Musset, tous interdits. À l'âge de dix-huit ans, de la même façon - et il faut comprendre qu'il n'y a pas beaucoup de jeunes de dix-huit ans qui fréquentent les bibliothèques à l'époque -, je me présente à la Bibliothèque de la Ville de Montréal et je demande tout bonnement le Génie du christianisme de Chateaubriand. J'ai failli me faire foudroyer! C'était quelque chose d'abominable car le livre était à l'index, dans l'enfer... C'était incroyable. Pourtant, plus on empêche quelqu'un, plus on tente de le brimer, et plus le contraire se produit; on se dit: s'il y a quelque chose à cacher... cela doit valoir la peine d'être découvert.

J'ai donc commencé à lire très tôt, c'est pour ça que j’ai lu plus de 25000 livres dans ma vie, et j'ai été heureux de connaitre à cette époque deux des plus grands libraires de Montréal: M. Pony, de la rue Sainte-Catherine, et M. Déom. Alors ces deux messieurs, braves messieurs, vieux messieurs, bons catholiques pratiquants, me procuraient des ouvrages sous le comptoir. Et de temps en temps, je pouvais lire certains titres et auteurs dits "érotiques "... comme Paul Féval!

Dans les années soixante, j'ai commencé à aller en Europe, occupant de très hautes fonctions sur le plan international comme délégué de sociétés professionnelles du livre à la Foire internationale du livre de Francfort, ou encore délégué général pour le Canada de l'Association des écrivains d'expression française. J'ai été le premier Canadien à aller à Francfort et à côtoyer le milieu de l'édition internationale! Et j'ai eu la chance inespérée de faire connaissance avec les plus grands éditeurs français ainsi que du monde entier. Je suis devenu un intime de Régine Deforges, de Jean-Jacques Pauvert, de même que du fameux Tchou, qui a publié des œuvres érotiques extraordinaires. Je suis entré chez Gallimard, j'ai rencontré Aragon, dont j'ai une cuvre tirée à seulement vingt-six exemplaires, Georges Bataille, André Pieyre de Mandiargues et puis Louis Pauwels, que j'ai fait venir au Québec pour la première fois comme conférencier. J'ai commencé à lire un certain nombre d'ouvrages, entre autres les œuvres de Romi (Mythologie du sein, Maisons closes, etc.) et j'ai découvert petit à petit ce qu'est l'érotisme.

V. \& I. - Mais entre les livres sous le comptoir et l'Europe, il y a toutes les années 50 pendant lesquelles vous avez milité au niveau municipal ainsi que dans des comités de moralité publique.

J.-Z.-L. Patenaude - J'ai été en effet étroitement lié à la Ligue d'action civique, parti politique municipal, de même que secrétaire-trésorier fondateur du Comité de moralité publique (1950-1960), président de la Fédération des Ligues du SacréCœur de Montréal et du Service de tempérance du diocèse de Montréal. Cela m'a amené à devenir le vice-président de l'Union internationale pour la protection de la moralité publique (UIMP) à Paris, et même chargé des relations de cet organisme avec le Conseil économique et social des Nations-Unies. Jai été, de plus, l'un des initiateurs de l'enquête sur la moralité, sur le jeu et le vice commercialisés à Montréal, enquête qui a duré quatre ans. Tout un volet touchait à la prosti- 
tution; cela m'a amené à me poser certaines questions au sujet de la moralité publique et politique, et puis ça m'a permis d'aller en Europe.

V. \& I. - Cela permet de comprendre certains titres que l'on retrouve dans votre fonds, des pamphlets tels que Contre la prostitution: réglementation ou abolitionnisme (1945), Faut-il tolérer la prostitution? (1953) ou encore des cathéchismes comme le Cathéchisme du mariage chrétien (1942).

J.-Z.L. Patenaude - Le contenu de la collection est lié à mes activités et à mes engagements. Rien n'a été planifié. Ainsi, une grande partie de mes livres, surtout dans le domaine de l'érotisme, je l'ai reçue en cadeau. Les grands éditeurs de Londres, d'Allemagne, m'en ont offert, gratuitement; c'est pour ça qu'on retrouve des ouvrages tirés d'éditions privées. C'est pour ça aussi que de nombreux livres sont dédicacés par les auteurs. Et certaines dédicaces sont fameuses. Régine Deforges m'en a fait une extraordinaire d'un ouvrage qu'elle avait édité. Elle avait écrit au début de l'Anglais décrit dans le château fermé, attribué alors à Pierre Morion: L'auteur gardant l'anonymat (comme vous le savez notre ami Mandiargues), c'est l'éditeur de ce livre, cher Léon Patenaude, qui vous le dédicace en souvenir d'une brève rencontre à Paris. Régine Deforges. Or, personne ne savait à l'époque qu'André Pieyre de Mandiargues en était l'auteur! Cela se passait en 1973 et on peut voir cette dédicace dans ma collection d'autographes et de signatures d'écrivains français et québécois, qui se trouve à la Bibliothèque municipale de la ville de Longueuil.

En 1955, je suis à Paris avec Gérard Pelletier et on déambule sur les quais. Il y a un livre qui vient de paraître, Histoire d'O de Pauline Réage, édité par JeanJacques Pauvert (j'ai l'édition originale, ça c'est rare!). Pelletier me dit: Léon, ça c'est un livre pour toi. On l'achète tous les deux... Pelletier était bouleversé. Moi, j'étais à Paris pour quinze jours; je regarde dans l'annuaire téléphonique et j'appelle un monsieur Pauvert à Sceaux. Un jeune homme me répond, je lui dis que je suis un Canadien de passage à Paris, que j'ai lu le livre, etc. Il me dit: Je serai à Paris tel jour; alors je linvite à venir prendre un verre à mon hôtel. Et je rencontre Jean-Jacques Pauvert. C'était téméraire, mais j’ai toujours été comme ça.

V. \& I. - Quels sont vos ouvrages favoris?

J.-Z.-L. Patenaude - Un des plus beaux textes érotiques qui existe en langue française, c'est "La nuit de Tolède» de Robert Brasillach, qui a été fusillé en 1945. C'est un épisode, le troisième, de Comme le temps passe... Et puis, il y a des ouvrages dans ma collection qui sont uniques ou presque. Il y a un Aragon à vingt-six exemplaires (Irène), $\mathbf{X}$ de Michel Beaulieu publié à dix exemplaires. Je vous signale aussi un petit livre - et vous pourriez même vous demander qu'estce que ça fait dans une collection comme celle-là? - un catéchisme publié par un de mes amis, j'ai nommé Martineau, un tout petit éditeur français, auquel j'étais très lié. Cet ami a publié le catéchisme du confesseur de la reine d'Espagne (les Mystères du confessionnal: manuel secret $d u$ confesseur de $M^{g r}$ JeanBaptiste Bouvier, 1968). Il faut lire ça! C'est le livre le plus pornographique que vous aurez lu de votre vie...

Du côté québécois, il y a le premier roman de Roger Fournier publié aux éditions Denoël, à Paris, dans les années 60. Mais il faut faire attention, ce roman 
est inconnu au Québec. Il n'est pas répertorié comme un roman québécois, mais comme un roman français ${ }^{3}$. Fournier ne pouvait pas publier cela au Québec, c'était trop érotique. Il était venu me voir et je lui avais dit: Je vais t'établir quelques contacts en Europe. Il a trouvé preneur chez Denoël. C'est un bon roman; en tout cas, ça me plaisait. Pour le nu, il y a mon ami Jacques de Roussan, le critique littéraire, qui publie en 1982 le premier album de l'histoire du nu au Québec: le Nu dans l'art au Québec. Je ne sais pas si vous l'avez vu, c'est un livre absolument impeccable. Et il y aussi Agaguk, le roman de mon vieil ami Yves Thériault, maintenant décédé.

V. \& I. - Mais est-ce vraiment de l'érotisme? Est-ce qu'il y en a eu au Québec?

J.-Z.-L. Patenaude - Jai mentionné le Fournier, mais il y a aussi un livre assez extraordinaire qui a été publié au Québec en 1970. C'est l'Homme qui se cherchait un fils, édité par le Cercle du livre de France. C'était un sujet osé pour l'époque: un gars qui fait une enquête sur les gais. Le livre n'a pas été retiré du marché mais... Alors, attention! Le livre n'était pas signé du nom de l'auteur! Ce qu'on pouvait lire, c'était un nom d'emprunt: François Brunante. Eh bien moi, je vais vous donner le nom de l'auteur! L'auteur, son nom m'échappe, c'est ce danseur, cet artiste très connu, qui a aussi publié une histoire des Grands Ballets canadiens aux Éditions du Jour, avec Jacques Hébert. Ça me revient, c'est Roland Lorrain ${ }^{4}$. C'était un livre assez extraordinaire...

Il y a eu quelques autres ouvrages où l'on pouvait sentir un peu d'érotisme. Il y avait cet ouvrage que j'aimais bien de Philippe Panneton/Ringuet, Fausse Monnaie (1947). Quand on connaissait Ringuet... vieux franc-maçon... En fait, à cette époque, il ne pouvait pas y avoir beaucoup de choses d'écrites ou de publiées; un certain nombre l'ont peut-être été sous le manteau et puis on les a perdues... Vous allez peut-être trouver ici et là des écrivains, ils n'étaient pas nombreux; c'était un sujet tabou au Québec. On dit qu'Arthur Buies aurait écrit des érotiques. Mais moi je serais surpris : avec sa sensibilité... André Malraux a écrit, lui, de très beaux érotiques sous des noms d'emprunt, quand il était avec Clara. Malraux n'était pas riche; il passait par Paris et il donnait des textes. Moi, si j'étais un éditeur français - j'en ai déjà parlé à quelques-uns - j'essayerais de voir sil n'y a pas moyen de publier quelques-uns de ces textes.

V. \& I. - Vous-même, vous n'en avez commis aucun?

J.-Z.-L. Patenaude - Non. Moi, j’ai écrit un ouvrage contre Jean Drapeau, le maire de Montréal. J'en ai eu assez dans ma vie d'avoir écrit ça. Jétais un homme d'action, un batailleur; un homme de liberté... pas un écrivain.

V. \& I. - Est-ce qu'il y avait un marché de l'érotisme au Québec?

J.-Z-L. Patenaude - Le marché de l'érotisme, on peut dire qu'il y a eu un tout petit marché d'ouvrages érotiques entre les deux guerres, grâce aux étudiants

3 A vrai dire, il n'est pas répertorié du tout.

4 Ce que confirme Denis Carrier dans le Dictionnaire des œuvres littéraires du Québec, tome V, p. 400-401. L'ouvrage de Roland Lorrain, paru aux Éditions du Jour, est intitulé les Grands ballets canadiens ou Cette femme qui nous fit danser, 1973. 
canadiens qui allaient à Paris... et aux médecins. Dans le domaine de l'érotisme, une classe sociale a toujours été bien représentée, ce sont les médecins! Il y a donc le Crapouillot, quelques grands romans; les étudiants les achetaient à Paris, les rapportaient ici et les mettaient dans leur bibliothèque. Après la guerre, un certain nombre d'intellectuels commencent à s'intéresser à cette question. Mais ce qui est très curieux, c'est que dans les années 70 les gens délaissent les textes et se mettent à collectionner surtout les livres d'art. Ce sont des beaux livres; les médecins en sont très friands, encore une fois. Je n'ai rien contre le livre d'art, il faut se comprendre - il y a de très beaux albums, à des prix abordables et qui sont très bien faits -, mais je connais des gens qui ont une bibliothèque de livres d'art absolument invraisemblable et qui n'ont jamais lu les livres qu'elle contient.

Les gens se mettent à collectionner des livres érotiques, mais ils le font en cachette. Tbut simplement parce qu'on ne veut pas que ce soit su, on ne veut pas passer pour un marginal.

V. \& I. - Mais, votre propre collection, était-elle connue?

J.-Z.-L. Patenaude - Oui! Il s'est produit un événement, à la fin des années 60 , qui m'a causé beaucoup d'ennuis et d'embarras. Réginald Hamel, de l'Université de Montréal, vient me voir et il me dit: Léon, est-ce qu'on pourrait faire l'inventaire de ta bibliothèque érotique? C'était pour son cours. J'ai dit oui, car je n'avais pas les moyens de le faire moi-même. Il a donc fait l'inventaire et m'en a donné une copie. C'était une grande bibliographie dans laquelle les titres de ma collection étaient identifiés. Il faut dire que déjà à cette époque on chuchotait sous le manteau: les collections cochonnes de Patenaude; les livres pornographiques de Patenaude; et le clergé disait: C'est un être abominable!

Six mois après, ç’a été un déferlement! Jai commencé à recevoir des téléphones et des visites d'étudiants et de professeurs chez moi, qui sonnaient à ma porte pour voir ma collection. Jai dit aux gens: Qu'est-ce que c'est que cette histoire? Je ne suis pas une bibliothèque publique! Réginald Hamel avait tiré sa bibliographie à cinq mille exemplaires, sans me demander la permission. Ça m'a causé beaucoup d'embarras, sur le plan éthique. C'est que ce n'était pas disponible! Je ne refusais pas à certaines personnes d'avoir accès à ma bibliothèque; les premiers qui y ont eu accès sont mes enfants, dès l'âge de seize ou dix-huit ans. Mais il faut un contrôle quand on prête des livres! Et puis, j'avais une collection assez complète pour l'époque. Evidemment, le Tout-Montréal et tout le Québec a su ça.

V. \& I. - Pourquoi avoir donné votre collection à l'UQAM?

J.-Z.-L. Patenaude - D'abord, il faut dire que donner mes archives, mes bibliothèques, ma collection, tout cela a pris cinq ans! Sans compter les deux années qui on été nécessaires pour tout répertorier. La première chose que j'ai faite a été de me demander à qui donner cela. La Bibliothèque nationale du Québec n'était pas intéressée, alors je suis allé voir les gens de l'UQAM. Je suis un vieil ami d'Hubert Perron, le directeur général de la bibliothèque. Je lui ai dit que javais deux grandes collections, uniques au Canada: l'une sur l'érotisme et l'autre sur la franc- 
maçonnerie ou les sociétés secrètes. Il m'a dit: Oui, ça nous intéresse. Alors là, des gens de la bibliothèque sont venus passer un mois à Hull où j'étais, car je travaillais en tant qu'éditeur du gouvernement du Canada, et ils ont fait l'inventaire de mes livres. J'ai décidé de donner ça à l'UQAM, pour trois raisons: premièrement, je suis pour la laïcité, la liberté de conscience, et l'UQAM est pour moi une université laïque; deuxièmement, à l'époque, c'était la seule université où il y avait un module de sexologie. L'érotisme, ça doit servir en sexologie! Troisièmement, j’ai toujours cru que je n'avais été que le fiduciaire de ma collection, que ce n'était pas à moi de la conserver, mais que ça appartenait plutôt au patrimoine littéraire et à la culture québécoise. Je l'ai donc léguée à mes compatriotes; et, surtout, parce que dans tous les domaines, même dans le domaine littéraire, on a évacué l'érotisme.

Cette collection est quand même unique. Quand j’ai fait mon don à la bibliothèque, on a fait une petite recherche pour savoir si une autre collection n'existait pas ailleurs au Québec. Et on n'a rien trouvé. Il se peut qu'il y en ait une autre, mais on ne l'a pas trouvée. Quelqu'un qui fait une recherche sur l'érotisme à cette bibliothèque et qui n'y trouve pas ce qu'il cherche peut se dire qu'il ne trouvera rien ailleurs au Québec. Je voulais que mon fonds serve aux étudiants, aux professeurs, aux chercheurs, à tout le monde. Aujourd'hui, on peut la consulter facilement, c'est sur BADADUQ (le système informatisé de la bibliothèque); c'est facile d'accès pour ceux qui le savent. Bien sûr, on ne peut pas laisser sortir les livres, qui sont dans la section des livres rares, mais il ne peut pas y avoir de censure, aucune limite.

V. \& I. - Parlez d'érotisme et de pornographie, et rapidement, on voit apparaître la possibilité d'une censure. Vous avez été témoin, je crois, ou sinon même lié à certains événements? Par le monde de l'édition, les salons du livre?

J.-Z.-L. Patenaude - Jai fait mieux que ça, j'ai préparé un texte sur la censure. Il y a deux ans, je ne me souviens plus à l'occasion de quel événement, Michel Roy de la Presse m'a téléphoné et m'a dit: Léon, est-ce que vous seriez prêt à me faire une petite histoire de la censure au Québec? Je lui ai dit: Laissez-moi vingt-quatre heures et j'envoie le texte! Qu'est-ce que vous voulez, c'est toute une partie de ma vie. Les documents, je les ais; il s'agit de les expliquer... Il m'a proposé une demipage dans la Presse. J'ai commencé à rédiger; le lendemain, je téléphone pour lui demander si ça marche toujours. Il me dit: Oui, oui. Je dresse mon plan - ce n'est pas facile de résumer, de condenser, d'expliquer aux gens ce qui s'est passé. Javais une question à poser à Michel, je lui téléphone; sa secrétaire me dit qu'il est très pris, qu'il ne peut pas me parler. Et j'apprends quil démissionne de la Presse!!! Alors l'article n'a jamais été rédigé; c'est pas le Devoir qui l'aurait publié!

Des principales affaires auxquelles j’ai été plus qu'intimement mêlé, il y a eu l'affaire Pellan. Là, on parle non seulement de censure mais aussi d'érotisme. Il s'agit d'un tableau d'Alfred Pellan qui était exposé dans le hall de l'hôtel de ville de Montréal. J'étais alors le secrétaire de la galerie. J'avais participé à la fondation de la Ligue d'action civique; j'avais fondé la ligue de la censure; j'avais mis Drapeau au pouvoir lors des élections de 1954. À un moment donné, quelquesuns de nos conseillers municipaux, plus catholiques que le pape et puis de tout ce que vous voudrez, décident de demander à M. Drapeau de retirer le tableau dit 
pornographique. Ce pauvre Drapeau, qui n'est pas fou - je parle du politicien -, pour plaire à ses conseillers, aux citoyens de l'est de Montréal, au cardinal Léger, etc., décroche le tableau. Je bondis chez Drapeau; je lui dis: Vous allez le raccrocher! Je n'ai peut-être pas d'ordre à donner au maire de Montréal mais je vous dénonce! Je venais de le faire élire... Alors Drapeau commence à réfléchir au geste qu'il avait posé. Tous les artistes contre vous, tous les écrivains... Quand même, $M$. Drapeau! Et je vais voir Pierre Desmarais, président du Comité exécutif du parti et je lui dis: Vous allez faire une déclaration; c'est vous le président de l'exécutif, vous allez donner l'ordre qu'on raccroche le tableau, c'est pas à Drapeau de donner l'ordre, c'est à vous! Desmarais fait une déclaration dithyrambique pour qu'on raccroche le tableau... Et on raccroche le tableau.

Un débat virulent s'engage. Moi, à ce moment-là, je suis propriétaire du journal Vrai, Jacques Hébert en est le directeur. Au début du débat, je dis à Hébert: Il faut porter un coup parce que c'est le journal du parti, un coup dur puis donner une leçon à Drapeau et même au parti; il faut franchir un pas, un gros pas! Je dis à Hébert: Tu vas aller ramasser des opinions à l'École des Beaux-Arts et ailleurs, et tu vas écrire un article, un article sur le fond de la question: la censure. Il me dit qu'il faudrait bien illustrer ça. Bonne idée, Jacques; demain matin, je t'apporte les illustrations. Alors, je fouille dans toutes mes collections, dans ce que j'avais, et j'apporte à Hébert la scène de la création d'Adam, de la chapelle Sixtine. Adam, la quéquette à l'air! C'était la première fois au Québec qu'un nu d'homme était publié intégralement en première page d'un journal! Toute la première page du journal Vrai. Et à l'intérieur du journal, il y avait le martyr de saint Sébastien et une autre photo. Trois en tout. On tirait quand même à vingt mille exemplaires! Imaginez l'impact!

Plus tard, il y a eu toute l'histoire des ballets africains, en 1967, le spectacle qui a été censuré parce que les seins, ça bougeait.

V. \& I. - Mais celle-là on la connaît; c'est un des cas de censure les plus drôles, du moins avec le recul.

J.-Z-L. Patenaude - J'ai mieux que ça! Jai un document extraordinaire qui n'a jamais été publié. Écoutez bien ça, vous allez voir d'où on part au Québec. Ça vient du fonds du cardinal Léger; ça date du 29 décembre 1959, ce n'est pas loin, ça. Paul-Émile Léger, cardinal prêtre de la Sainte Église Romaine, etc. En vertu des pouvoirs à Nous concédés par le Saint-Siège à cette date, Vu les Motifs à Nous exposés: Nous autorisons M. Georges-Étienne Cartier, M.D. - c'est l'arrière-petitneveu de Sir George-Étienne Cartier, le directeur médical, nul autre, de l'HôtelDieu de Montréal, l'une des plus grandes sommités médicales qu'on ait eues au Canada, un homme lettré, ayant étudié en Europe - pour une période de trois années, à dater de ce jour, à lire les ouvrages à l'index et à les garder, mais en les plaçant dans un lieu sûr, à l'abri de toute indiscrétion. Vous ne rêvez pas là, c'est un document que je vous donne! Sont exceptés de cette permission les livres qui traitent de sujets immoraux ou qui, ex professo, attaquent la religion. Le bénéficiaire de ce rescrit devra toujours, avant de lire un livre à l'index - en 1959!!! —, prendre l'avis d'un confesseur prudent. Et là, il y a un astérisque: quelques romans de Balzac; Madame Bovary de Flaubert; le Rouge et le Noir de 
Stendhal; les Provinciales de Pascal et les Essais de Montaigne. Et c'est signé: Paul-Émile Léger. Vous vous imaginez!

Je vous donne une copie du document, mais j'ai l'impreșsion qu'll ne va pas aimer ça. Nous ne sommes pas sur la même longueur d'ondes, depuis longtemps.

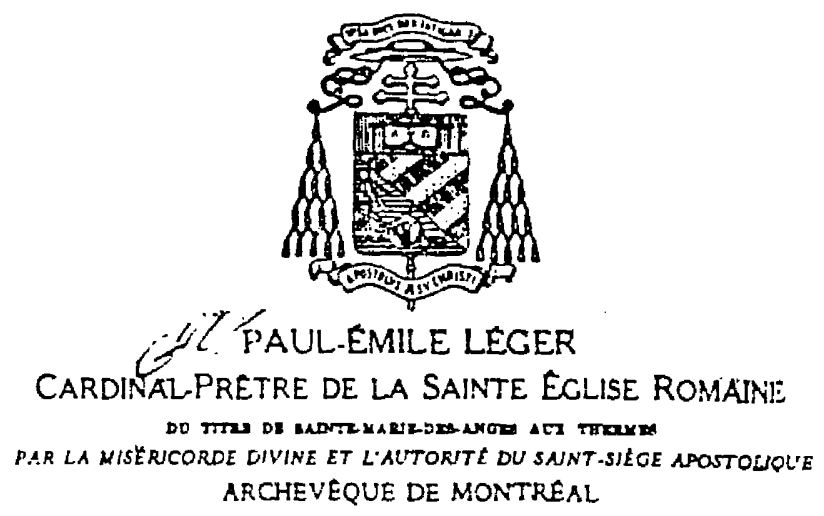

En vertu des pouvoirs à Nous concédés par le Saint-Siège, le

Vu les motife a Nous exposés:

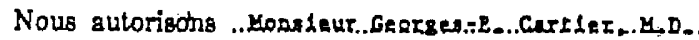

pour une période de truls années à dater de ce jour, à lire les ouvrages ì l'index’et à les garder, mais en les piaçant dans un lieu sar, à l'abri de toute indiscrétion.

Sont exceptés de cette permission les livres qul traitent de sujels anmoraux ou quil, ex protesso, attaquent ia religion.

Le bénéficiaixe de ce rescrit devrs coujours, avant de lire un lirte \&े l'index; pren. dre l'aris d'un confesseur prudent.

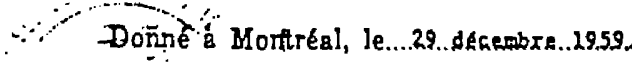

* qualques rosine de Melac;

Hadane Bovary de Plaubert; te

rouge et le nolr de stendhal;

Lee Provinclalia. de pescal et

Le Resalo de Montalgae.

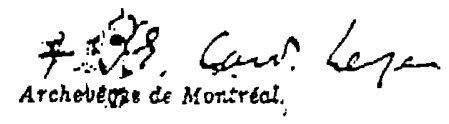

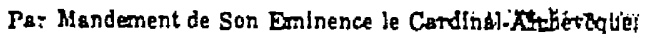

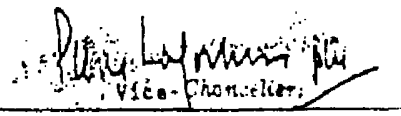

V. \& I. - De votre histoire de la censure, il reste encore des épisodes, n'est-ce pas?

J.-Zn-L. Patenaude - Il s'est passé quelque chose en 1963, lors du Salon du livre de Montréal que jorganisais. On avait exposé une scuplture de Robert Roussil qui a fait scandale. Avec tout ce bon peuple qui vient voir des livres, je dis 
à Hébert: Il faut frapper un gros coup; il va y avoir bien des religieux, des frères, des sœurs... - d'ailleurs le cardinal Léger est venu me décorer! - il faut frapper un coup, que je lui dis. Tu vas faire une exposition à ton kiosque des Éditions du Jour. Présenter une chose qui n'a jamais été montrée. C'était la Famille de Roussil. C'était très érotique; enfin, pour 1963 ! Et on a payé Roussil en plus, cent ou deux cents dollars. Il n'était pas habitué! Je lui avais expliqué: Nous, au Salon du livre, on est là pour montrer des livres; toi, tu vas venir exposer. Mais le plus drôle, ça été quand Pierre Laporte est venu inaugurer le Salon. Vous savez, on a été des intimes toute notre vie: j’ai été un de ses organisateurs, son trésorier politique, etc. Il était alors ministre des Affaires culturelles et je lui ai dit: Viens inaugurer le Salon du livre. Pierre Laporte s'amène donc là en triomphant, et le premier kiosque à visiter en entrant, c'est celui des Éditions du Jour, où l'on avait déposé le Roussil. Pierre Laporte visite tout ça, il voit la sculpture et là, en entrevue, il explique comment il admire ce Roussil. Or, dans les journaux, il n'y avait que des lettres de protestation! Pauvre Pierre!

C'était audacieux, c'était défier la censure. Aux Éditions du Jour, dont jétais le secrétaire-trésorier à l'époque, on a aussi publié le premier album de nus au Québec. Et c'était en 1969 ! Jacques Hébert vient me voir — on était presque des frères siamois! on s'entendait tellement bien - et il me dit: J'ai un livre sensationnel à publier mais financièrement... ça va en demander. Je réponds: Ce n'est pas un problème, l'argent, on en trouvera. Et on l'a fait. C'était le Corps secret de Mia et Klaus, les photographes. Un album de nus avec des poèmes de Jacques Brault. Il n'y avait jamais eu, à ce moment-là, d'album de nus publié au Québec, jamais!

Ensuite, il y a eu, bien sûr, l'affaire Régine Deforges. Jai fait scandale avec elle un jour à Nice: elle portait une robe qui n'était pas décolletée, au contraire elle n'avait qu'un devant... une très belle robe de soirée! Je devais inaugurer le bal et je décide de le faire avec Régine. Il y avait quatre-vingts musiciens, des photographes, beaucoup de monde. Le lendemain, on a fait la première page de tous les journaux de Nice. J'ai pas besoin de vous dire que quand la photo est arrivée au Québec, M. Guy Frégault, sous-ministre des Affaires culturelles m'a dit: $M$. Patenaude, comment osez-vous? Je lui ai répondu: $M$. Frégault, après minuit, je fais ce que je veux, ça ne vous regarde pas.

L'affaire Régine Desforges est un cas important de censure. Elle avait été emprisonnée en 1973 pour avoir refusé de se censurer. Elle devait déposer à la police des ouvrages, qui devaient être édités à sa maison d'édition l'Or du temps, pour contrôle préalable. Elle a refusé et elle a été condamnée ${ }^{5}$. Alors moi, j’ai pris l'initiative de faire une pétition et de ramasser de l'argent pour la sortir de prison. Ça se passait en France, mais ç'a eu des répercussions au Canada, parce qu'on était à une époque où le gouvernement fédéral voulait développer davantage le code criminel.

Et il ne faut pas oublier dans tout ça l'affaire Guy Delorme. Un autre cas de censure avec saisie de livres! Mais québécois cette fois. Saisir des livres... c'est

5 Après saisie de cinq publications par la police et six inculpations pour outrage aux bonnes mœurs par la voie du livre. 
bien beau quand c'est du passé, quand ça n'existe plus... on oublie comment ça s'est réglé, on oublie qu'il a fallu des gens courageux, qu'il a fallu mener des batailles. La fameuse affaire Delorme; j’y ai été mêlé directement: les livres qui ont été saisis m'étaient destinés. Guy Delorme était un brave libraire de la Place Ville-Marie; il me faisait venir des livres, les collections de Pauvert, certains beaux albums. Un jour, toute une collection des ouvrages de Sade a été saisie par les fonctionnaires de la douane. Ils avaient une liste et ils prenaient tout ce quils pouvaient trouver. Le premier procès, dans l'ordre chronologique, fut celui d'Histoire d'O de Pauline Réage. J'ai dans ma collection l'édition illustrée par Léonor Fini (Tchou, 1968). Et ce pauvre Delorme a été condamné, au nom de la morale, à payer $200 \$$ d'amende. Les éditeurs ont mis la main dans leur poche... mais quand même.

V. \& I. - Ce jugement, c'est celui du juge Anctil, n'est-ce pas?

\section{J.-Z.-L. Patenaude - C'est ça, vous l'avez.}

V. \& I. - Je l'ai eu par le livre de Jacques Hébert, Obscénité et liberté.

\section{J.-Z.-L. Patenaude - Vous avez vu à qui le livre est dédié?}

V. \& I. - C'est même la première chose que j'ai vue: À J.-Z.-Léon Patenaude, ami des livres et de la liberté! Le jugement est clair: [...] la Cour est d'opinion que la caractéristique dominante d'Histoire d'O est l'exploitation indue des choses sexuelles ou la description de scènes de cruauté et de violence continuellement répétées de sorte que même si l'auteur avait voulu démontrer symboliquement la supériorité de l'âme sur le corps il devait livrer son message dans des termes acceptables pour la morale publique. La morale publique ne peut être sacrifiée au progrès. En conséquence la Cour maintient l'accusation telle que portée et ordonne la confiscation de l'ouvrage [...]. ${ }^{6}$

J.-Z.-L. Patenaude - Mais il y a une suite! Quand le procès fut terminé, je me suis mis à réclamer du tribunal qu'on me remette mon livre! Un jour, je suis même allé voir le greffier du juge et je lui ai dit: Vous, vous l'avez lu pendant le procès, le juge, lui, l'a gardé dans sa bibliothèque, mais ce n'est pas à lui. Dites donc au juge qu'il me le redonne. Tbute la cour a lu ce livre-là, je le sais, je peux vous nommer mes amis juges qui ont lu le livre. Comme c'est le seul exemplaire qui a été saisi, demandez au juge d'être assez honnête et de me remettre mon livre. Is ne me l'ont jamais remis!

V. \& I. - Faites-vous une distinction entre ce qui est de l'érotisme et ce qui est de la pornographie?

J.-Z-L. Patenaude - Oui, oui, il y a une distinction à faire, mais une distinction très subtile, qui se retrouve moins dans l'écrit ou dans la littérature que dans le visuel. Car l'érotisme, ce sont des mots... les mots qu'employaient les grands auteurs érotiques; des mots tels que la pudeur. Il y a quand même eu une période, au temps de Flaubert, où des grands écrivains ont écrit des choses merveilleuses. À la période romantique. Mais maintenant cela a disparu. Je pense que tout cela est lié à un phénomène apparu il y a trente-cinq ans, enfin, depuis les années 50 
ou 40 , c'est-à-dire la libération sexuelle chez la femme, qui a débouché sur le féminisme radical. Ce qui est arrivé est simple à résumer: on a évacué l'érotisme et ça a donné la pornographie. D'ailleurs, depuis quelques années, il y a bien des femmes qui ont commencé à s'en apercevoir. En se libérant, on a déchaîné les vieux démons qui étaient dans l'homme. Mais au moins, depuis quelques années, il y a une prise de conscience de ce qu'est vraiment la pornographie.

V. \& I. - Maintenant que vous avez donné votre, enfin, vos collections de livres et de documents, que faites-vous de votre temps?

J.-Z.-L. Patenaude - Mais je n'ai pas arrêté! Je participe aux activités du Cercle Condorcet à Montréal, dont je suis le trésorier et dont le président est Luc Alarie et le vice-président Jean-Paul De La Grave. Justement, le 23 mai (1989), on a reçu M. Claude Julien, du Monde diplomatique. L'année dernière (1988), j'ai aidé à créer "l'Ordre du Livre Ouvert", un ordre de mérite de l'édition. Je suis l'éditeur de la Fondation Lionel-Groulx. Je fais partie aussi du projet "Cultes", qui s'occupe des sectes religieuses et qui a été fondé pour déprogrammer les gens. Il y a, par exemple actuellement, un sérieux problème avec les témoins de Jéhovah, à Montréal. Il n'y a plus de liberté d'esprit, plus de liberté de conscience. Ils ne lisent pas, ils n'ont pas le droit de lire autre chose que la Bible... pas les journaux, rien. C'est absolument incroyable. C'est du lavage de cerveau! Mais qu'est-ce que vous voulez, les gens sont à la recherche du spirituel. Mais quand c'est cela qu'on leur offre, comment voulez-vous qu'ils trouvent une réponse à leurs problèmes? Souvent on cherche de trop grandes solutions. Moi, je l'ai toujours dit, je suis l'homme du "small is beautiful ". Sil y a un problème, on trouve des éléments de solution. On examine bien ce qui en est, on se sert de certaines notions, de certaines valeurs, de l'éthique, de la morale, des cultures, et on finit par trouver.

Si vous voulez résumer ma vie, vous pouvez dire que j’ai été un gars qui a dérangé tout le monde. Depuis l'âge de treize ans, je n'ai fait que déranger. Et je continue à déranger; je suis au mouvement laïque québécois, pour la laïcité et la déconfessionnalisation, la séparation de l'Église et de l'État. Ce ne sont pas des sujets populaires au Québec, même en 1989.

Je suis un homme de liberté, avec toute la prudence, la tolérance qu'il faut y mettre... et je le demeure encore aujourd'hui. On peut dire que rien ne m'a été étranger. On m'a déjà nommé dans les années 70, c'était Louis Martin qui l'avait fait, "M. Touche-à-tout»: Vous avez été, vous êtes, ou vous serez membre d'un organisme dont vous avez été, vous êtes, ou vous serez le secrétaire général! Bien sûr, on ne touche pas à tout d'une façon égale mais cela n'empêche rien. J'aimais ouvrir la voie. Je lançais des initiatives et, du moment que ça marchait, je m'en allais. Ce n'était pas ma propriété. C'est comme ça que j'ai fondé je ne sais pas combien d'organismes au Québec: la Ligue des Droits de l'Homme, la Société du droit d'auteur au Québec, les CEuvres de l'abbé Pierre au Canada, et ainsi de suite. La liste est longue. Je n'ai pas voulu écrire mes mémoires. À quoi ça peut servir? J'ai préparé un document assez complet où je raconte tout ça. Il est daté de juin 1985. On y retrouve l'histoire de ma vie: l'histoire de mes ancêtres d'abord; puis, celle de ma vie, de ma famille, de mes activités. Je vous le laisse; ça pourra vous servir. 
V. \& I. - J'en suis sûr. Une dernière question: si, à la fin des années 80 , vous étiez au début de votre vie, recommenceriez-vous la même collection?

J.-Z.-L. Patenaude - Je ne sais pas. Il y a deux choses de sûres: je n'ai ni regrets, ni remords. Donc, pas de si j'avais su. Si on avait su, on n'aurait pas pu faire ce qu'on a fait, car on aurait planifié autre chose! Mais recommencer... Dans le domaine de l'action, ce sont les circonstances qui nous forcent à agir. On ne décide jamais vraiment. Si javais étudié toutes les conséquences de mes actes... mais ça aurait servi à quoi ? Les conséquences sont ce qu'elles sont.

Dans ma vie, j'ai toujours eu une sainte horreur de l'argent, le veau d'or de Moïse! C'est utile, l'argent, on en a besoin, mais l'idée de posséder, l'appât du gain, ça n'a jamais été pour moi des préoccupations. J'ai souffert de ce manque de désir... mais cette attitude m'a assuré, je m'en suis rendu compte seulement ensuite, une grande indépendance et une grande liberté d'esprit. Voilà pourquoi j'ai toujours été un homme qui disait ce qu'il pensait; je n'ai jamais tué, je n'ai jamais cru posséder la vérité ni la main de Dieu... Oui, une grande indépendance. De faire valoir un point de vue, de défendre certaines idées, certaines opinions, ce qui n'est pas facile surtout auprès des pouvoirs, des puissants... On n'est pas là pour comptabiliser, pour compter, mais il n'en demeure pas moins que pour moi, l'argent n'a jamais été très important. Une autre valeur que j’ai traînée toute ma vie - je ne sais pas d'où elle vient, de mes parents, de l'école, mais je l'ai depuis au moins l'âge de quinze ans -, c'est que ce n'est pas le nombre d'années qu'on a vécues qui comptent, c'est ce qu'on a fait quand on vivait.

Montréal, le 25 mai 1989 
DOSSIER 179

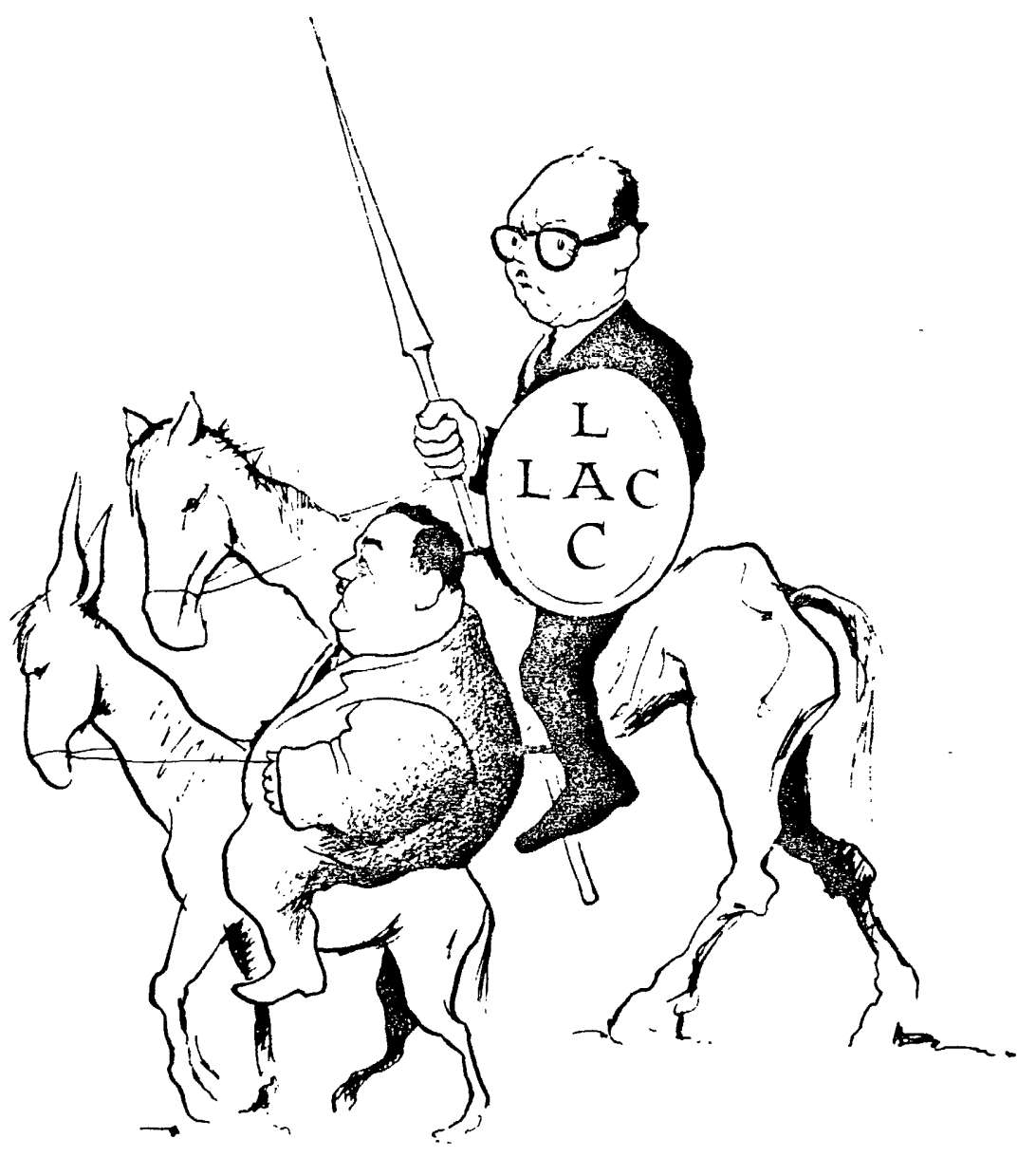

"À la recherche de Sarto [Fournier]*, caricature de Normand Hudon parue dans le Devoir du 30 septembre 1959, représentant J.-Z.-Léon Patenaude et le maire Jean Drapeau. Reproduite avec l'aimable autorisation du Devoir. 